\title{
Cautionary notes \\ on the undesirable secondary effects of lons term orthopedic and ventilation mask therapy in the treatment of OSAS
}

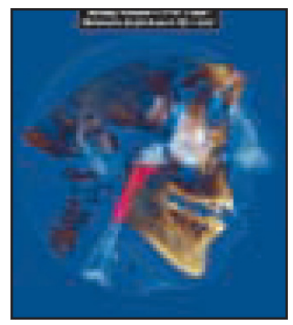

\author{
Manish VALIATHAN, Austin PHOENIX, Martin PALOMO, \\ Mark HANS
}

Traduction de Jacques TALMANT

\begin{abstract}
Snoring and Obstructive Sleep Apnea (OSA) are caused by repetitive dynamic closure of the oropharyngeal airway. There exists substantial and growing epidemiologic evidence that OSA is associated with a variety of systemic conditions, including but not limited to, systemic hypertension, stroke, congestive heart failure, increased motor vehicle accidents, impaired quality of living and mortality. There is an equal amount of literature on the use of dental appliances as a treatment modality for OSA. The object of this report is to review the literature in regards to three vital aspects of this disease: a) the etiology and sequela of OSA; b) the dental management of the condition and its mode of action; and lastly, c) the positive and negative outcomes/risks, of dental management.
\end{abstract}

\section{KEYWORDS}

Obstructive Sleep Apnea

Oral devices

Dental appliances

treatment protocols. 


\section{1 - INTRODUCTION: THE ETIOLOGY AND SEQUELA OF OSAS}

Sleep Disordered Breathing is a general term that covers conditions that are characterized by recurrent upper airway obstructions that result in episodes of asphyxia and interruption of normal sleep pattern's ${ }^{11}$. These conditions include chronic snoring, obstructive sleep hypopnea, and Obstructive Sleep Apnea Syndrome (OSAS). Patients with OSAS have daytime symptoms of sleepiness, inattention, and fatigue resulting from repetitive episodes of partial or complete airway obstruction lasting at least 10 seconds that cause a fall in blood oxygen saturation levels, rise in carbon dioxide levels, and an increased heart rate ${ }^{43}$. Most patients with the syndrome have 20-30 or more episodes per hour of sleep. Obstructive apnea must be differentiated from Central Apnea. In central apnea both airflow and respiratory effort are absent. In obstructive apnea, absence of airflow is accompanied by increased chest muscle movement that is detected on a typical polysomnogram.

The causes of obstructive sleep apnea are thought to be related to three major factors: airway geometry, lumen suction pressure and muscle tone ${ }^{45}$. Certain craniofacial anatomic factors may operate specifically to reduce airway patency ${ }^{3,9,29,30,31}$. In addition; obesity, alcohol use, and abnormalities in ventilatory control are risk factors of the disorder. Since all of these factors are subject to both genetic and environment influences, there may exist a significant familial basis for OSAS ${ }^{34,42}$. The narrower the airway, the more susceptible it is to closure and the more dependent it is on muscle tone to remain patent ${ }^{45}$. Several anatomical features contribute to the narrowing including enlarged adenoids and tonsils in children, a receding mandible resulting in reduced anteroposterior dimension of the oropharynx, enlarged tongue in obese individuals, and any neoplastic growth that may obstruct the lumen. Increased lumen suction pressure is created when the forces generated by inspiratory muscles produce greater positive transmural pressure favoring upper airway collapse. Increased resistance in the nasal airway, for example, will create greater suction pressure in the oropharynx. Finally, muscle tone influences upper airway patency. The tone of the dilator muscle of the oropharynx must remain high in order to maintain airway patency. Decreased muscle tone makes the airway more susceptible to collapse, and at sleep onset, there occurs a reduction in tonic as well as phasic muscle activation. Several muscles, including genioglossal muscles, tensor palati, and geniohyoid are thought to be important in maintaining upper airway patency.

Obstructive Sleep Apnea Syndrome can be considered a continuum that begins with snoring (mild to severe) and extends through the more significant disturbances of multiple apneas, hypersomnolence and the frequent nocturnal arousals ${ }^{38,44}$. Snoring (sounds produced by turbulent flow in a narrow or obstructed airway at the level ofnasopharynx or oropharynx) is the hallmark of the syndrome and is the most common reason for patients 
to contact an otolaryngologist ${ }^{1}$. Obstruction can occur at the oroor naso pharynx ${ }^{41}$. Wilms and coworkers [1982] studied 25 adult men diagnosed with the syndrome and found airway anomalies at the oropharynx level in $76 \%$, at the hypopharynx level in $44 \%$ and at the larynx level in $8 \%$ of cases $^{46}$. Some research studies attempted to distinguish features in non-apneic snorers from those in apneic snorers and provide a likely explanation as to why some snorers develop complete airway occlusion during sleep ${ }^{38}$. Stauffer and colleagues [1987] conducted a well controlled study that demonstrated that increased inspiratory resistance to air flow in the naso-oropharynx was present during wakefulness in overweight men with obstructive sleep apnea $^{38}$. Differences in craniofacial anatomic structures of apneic and non-apneic snorers in the upright and supine position have been reported by Pracharktam et al. ${ }^{30,31}$. This group concluded that, while the magnitude of some variables increases in the supine position, these increases produced no additional posture related differences between the two groups. Thus anatomic assessment of subjects at risk for sleep apnea can be made in the upright and awake state. The most consistent cephalometric findings related to OSAS are a lower position of the hyoid bone, increased length of the soft palate, and decreased size of the posterior pharyngeal space.

It is timely and vital to find treatments to alleviate sleep apnea in the light of life- threatening consequences of this disease. Adult apnea patients exhibit a multitude of cardiac symptoms. Systemic hypertension occurs in 40-60 percent of these patients, and its severity depends on the severity of apnea $^{11}$. Systemic blood pressure increases by as much as $20 \%$ immediately after apneic episode termination. Several mechanisms have been proposed for this phenomenon, including hypoxia, acidosis, increased respiratory effort, and the increased sympathetic activity associated with the awakening. Cardiac output also decreases during apnea because of decrease in heart rate without concurrent increase in stroke volume ${ }^{40}$. Furthermore, in OSA patients combined effects of hypertension, hypoxia, and increased sympathetic activity can lead to pulmonary hypertension, cardiac arrhythmia, and ischemic heart disease ${ }^{11}$.

Obstructive Sleep Apnea significantly increases the risk of stroke due to decreased cerebral blood flow, and death ${ }^{47}$. In an observational cohort study of more than a thousand patients, patients with high apneahypopnea indices had a greater hazard ratio of developing a stroke and death from any cause. Ferguson ${ }^{11}$ agrees that five-year survival is greatly reduced in patients with untreated OSA, mainly due to cardiovascular complications. Clearly there exists a growing volume of epidemiological and experimental evidence that OSAS, and to a lesser degree snoring, are associated with a wide variety of adverse health outcomes and has been associated with coronary artery disease, stroke, congestive heart failure, atrial fibrillation, increased motor vehicle accident rate, sleepiness, impaired quality of life, and increased mortality ${ }^{18,37}$. Although several epidemiologic studies suggested a relationship between snoring and hypertension, 
cardiovascular disease, and cerebrovascular disease, most of these studies were not able to discern the difference between primary snoring and patients with a mild variant of OSAS. Nevertheless, snoring represents an important social problem, and contributes to impaired sleep quality of the bed partners of those who snore ${ }^{4}$.

In children, cognitive and behavioral abnormalities are associated with sleep- disordered breathing. Due to frequent awakening and intermittent hypoxia, daytime function can be impaired. According to ElAd et al. ${ }^{10}$, the most common abnormalities are decreased vigilance, depression, anxiety, and hyperactivity. In a study of 403 children Mulvaney et al. ${ }^{27}$ the Child Behavior Checklist was used to show that children with high Respiratory Disturbance Index showed greater aggression, social problems, inattention, and oppositional behavior. Many of these behavioral problems improved significantly after adenotonsillectomy procedures ${ }^{2,25}$. Cognitive dysfunction is commonly observed in children with sleep-disordered breathing $^{6}$. In one study, such children were 8 times more likely to develop cognitive problems compared to normal children. Academic performance often suffers in children with sleep-disordered breathing ${ }^{26}$. Gozal et al. ${ }^{14}$ stu- died over 1500 middle school children who were in the top $25 \%$ of their class and the bottom $25 \%$ of their class. Those students who had lower grades were more likely to have snored during early childhood than children with better academic performance. Just like the behavioral problems, cognitive performance improved in children who underwent adenotonsillectomy to treat obstructive sleep apnea ${ }^{26}$.

Sleep-Disordered Breathing is not an uncommon disorder. The prevalence of this life-threatening condition has been estimated to be as high as $24 \%$ in men and $9 \%$ in women ${ }^{11}$. The prevalence of snoring in children has been reported between 3.2 and $12.1 \%$, while the prevalence of Obstructive Sleep Apnea Syndrome ranged from $0.7 \%$ to $10.3 \%^{36}$. In a German study of 332 students between the ages of 11 and 15, 28\% reported either snoring, or insomnia, or nightmares. $15 \%$ of the children reported snoring accompanied by either insomnia or parasomnia (nightmares, sleepwalking $)^{19}$. In another study of orthodontic patients prevalence of habitual snoring was From this summary it should be noted that all forms of Sleep Disordered Breathing, ranging from snoring to OSAS, are found in children and adolescents.

\section{2 - COMMONLY PRESCRIBED TREATMENTS AND THEIR MECHANISMS OF ACTION}

Numerous treatment options, ranging from oral appliances and continuous positive airway pressure (CPAP) to surgery, are available to patients with sleep apnea. These options vary widely in regards to patient acceptance and effectiveness. A brief overview of the treatment alternatives will 
be provided here, with greater emphasis placed on orthodontic appliances.

Although there are a variety of dental devices to "treat" OSA, they tend to differ in appearance more than function. The underlying principle of these devices is to increase pharyngeal airway volume by holding the mandible in a protruded position. The objective for these appliances in patients with primary snoring without features of OSAS or upper-airway resistance syndrome, is to reduce the snoring to a subjectively acceptable level. On the other hand, for patients with OSAS, the desired outcome of treatment includes the resolution of the clinical signs and symptoms of OSAS and the normalization of the apnea- hypopnea index and oxyhemoglobin saturation ${ }^{21}$. Guidelines from the American Academy of Sleep Medicine suggest that oral appliances are appropriate for use in patients with primary snoring who do not respond to or are not appropriate candidates for treatment with behavioral measures such as weight loss or sleep-position change ${ }^{21}$. While oral appliances may not be as efficacious as CPAP they can be successfully used for patients with mild to moderate OSAS who prefer dental devices to CPAP, or who do not respond to $\mathrm{CPAP}$, are not appropriate candidates for CPAP, or who fail treatment attempts with CPAP.

Many devices are adjustable allowing either the clinician or patient to advance the mandible based on the patient's response to the device. The response is often determined by subjective assessments of improvement in daytime sleepiness or bed partner reports of a decrease in snoring. It is important to note that a decrease in snoring may be the result of an increase in apneic activity rather than a decrease i.e. the patient has stopped snoring because he has stopped breathing. A post appliance polysomnogram would be the most reliable method to document device effectiveness.

Though titration of CPAP is routinely done during overnight polysomnography studies, there are no reports in the literature documenting the titration following the advancement of the mandible with a dental device during overnight polysomnography. As dental devices become more popular it is likely that such a titration device would be clinically useful.

The efficacy of increasing pharyngeal volume with mandibular protrusion has been documented and figure 1 and 2 show these changes on 3D conebeam computed tomographic images. Note that the increase in pharyngeal volume is quite dramatic. Similar changes in airway have been shown on cephalometric radiographs. Hans et $a l^{16}$ designed a study to evaluate the effectiveness of two intraoral devices in reducing the $\mathrm{Re}$ spiratory Disturbance Index (RDI) and Epworth Sleepiness Scale (ESS) scores in a group of 24 adult volunteers with a history of loud snoring. Subjects were randomly assigned to two groups. Twelve subjects were fitted with a dental device designed to increase vertical dimension and protrude the mandible (device A). The other 12 subjects received a different device designed to minimally increase vertical opening without protruding the mandible (device B). RDI was recorded at two time periods: (TO) 


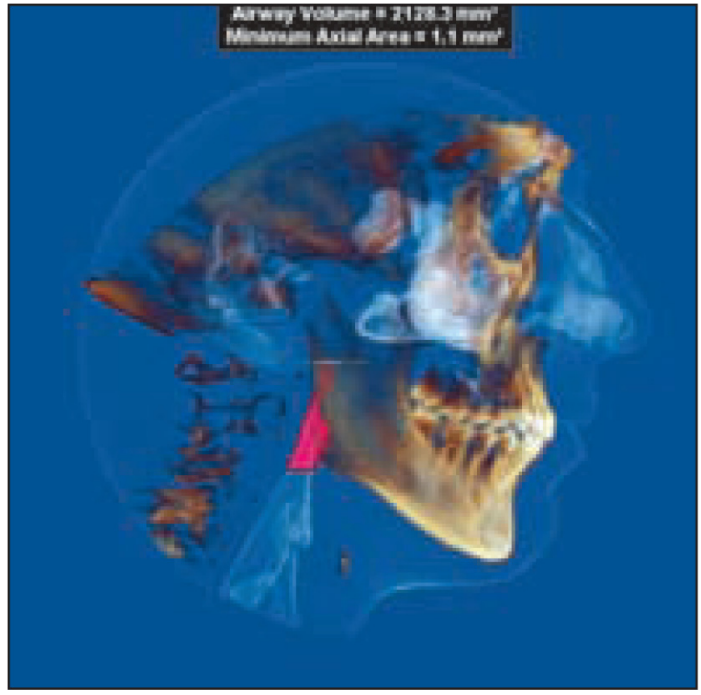

Figure 1

CBCT of an adult patient with OSAS without an oral appliance in place. Note the decreased in airway volume.

before using any dental device and (T1) while using a dental device 2 weeks after the initial delivery date. The mean RDI and ESS scores at TO and T1 were compared for subjects in the device A group and device group. The effectiveness of the two devices was estimated by comparing the difference in RDI scores from T0 to T1. Device A reduced RDI scores in 9 of 10 subjects who completed the study, with a mean reduction in RDI of $14.5(p<$ or $=0.05)$ and in ESS score of 3.8 ( $p<$ or $=0.005)$. Device $B$ showed no change or an increased RDI score in 8 of 8 subjects who completed the study demonstrating a decrease in anatomic risk factors when patients are wearing a protrusive device compared to a plecebo control.

The nasal airway is supported by bone and cartilage as is the trachea and bronchi. It is the pharyngeal

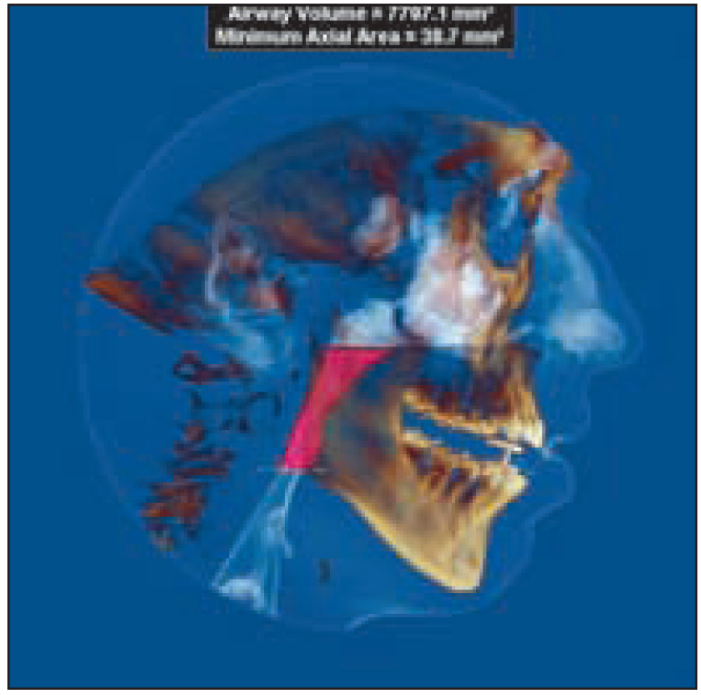

Figure 2

CBCT of an adult patient with OSAS with an oral appliance in place. Note the increase in airway volume.

airway that is largely dependent on muscular tension to remain patent. As seen in figures 3,4 and 5, the airway when modeled as a tube, can collapse due to an increase in resistance to airflow that exceeds the ability of the neuromuscular system to maintain the airway. Consider the air-way as a straw of a given size. When water is sucked through the straw the straw does not collapse. However, if a viscous liquid like a milkshake is sucked in through the straw the straw collapses. To prevent this collapse you can reduce the resistance or increase the straw lumen. CPAP decreases airway resistance by increasing the pressure inside the lumen of the airway whereas dental devices prevent collapse by increasing the size of pharyngeal airway. Ultimately obstructive apnea is a neuromuscular and not an anatomic disease. This is a critical distinction as dental practitioners tend 


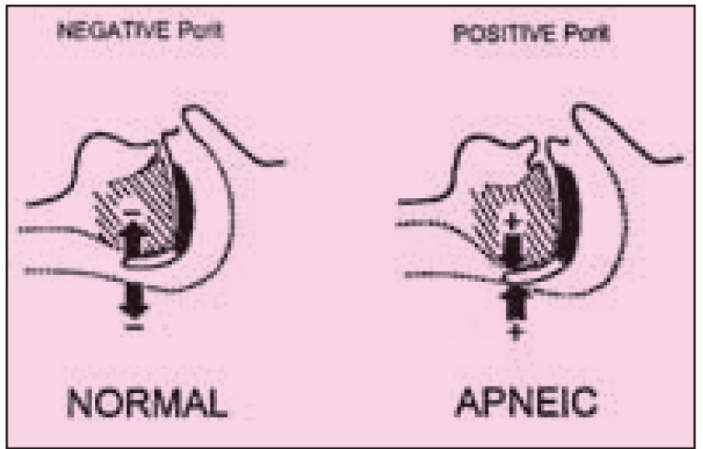

Figure 3

Showing the normal phyaryngeal airway with negative critical closing pressure (NEGATIVE Pcrit) compared to the apneic patient where closing pressure exceeds the ability of the musculature of the oropharyn to maintain a patent airway.

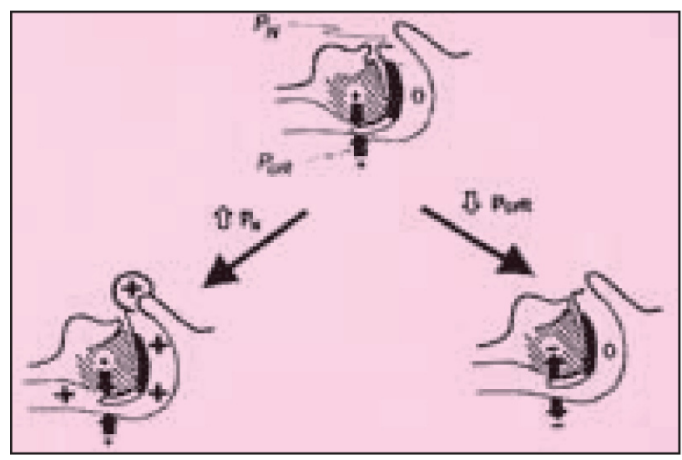

Figure 4

Showing that the collapsed airway can be expanded by increasing the pressure within the airway lumen $\left(P_{n}\right)$ as is done with CPAP or alternatively the lumen of the airway can be increased as is done with dental devices and bimaxillary advancement surgery.

to treat the symptoms with only a partial understanding of the etiology of OSAS. This becomes increasingly problematic as the legal risks of such treatments are likely to lead to claims of OSAS being caused by anatomic changes in the facial and dental structures. Such a claim was recently

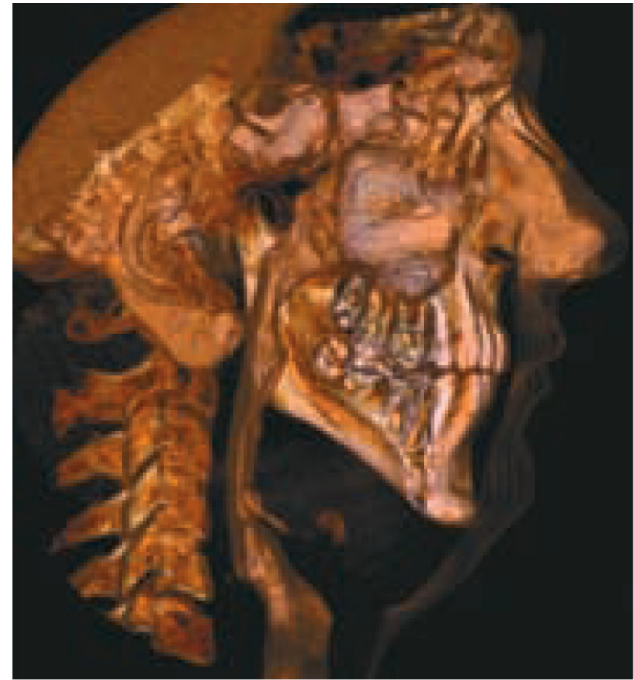

Figure 5

Patient treated with extraction of four first bicuspids referred to the Case Imaging Center to rule out anatomic constriction of the pharyngeal airway. Note the length of the airway and the potential impact of this length on the critical closing pressue. The size of the airway is normal.

brought to my attention by the following email.

Dear Doctor,

My daughter (now almost 21 years old) underwent multiple tooth extractions as a child for orthodontic reasons. She had somewhere in the order of 10 or so deciduous teeth pulled by the time she was 12. When she was in high school, she spent several years with unexplained fatigue, and was subsequently treated for depression. The depression meds led to Gl complications. After several years of not resolving the fatigue, we finally got a diagnosis of sleep apnea. She was treated with a Bipap machine, and made a very good recovery (including the 
depression and $\mathrm{Gl}$ issues) over 4 years.

As an adult, she came to feel that the Bi-pap machine was a disability, rather than a 'life-saving' device, and is in the process of learning to use a dental appliance. During her evaluation, we asked what could have caused her sleep apnea at such a young age. It was suggested that maybe all her tooth extractions could be the culprit. As a parent making those dental decisions for her child, I have been devastated by this. The possibility that I authorized procedures that will have life-long negative consequences for my daughter has been a lot to acknowledge. So, I am looking for some answers, and to raise flags so other families can be more understanding of the risks, if there truly are risks, of early multiple tooth extractions.

Do you know of any research that addresses this issue? Is there any general opinion among orthodontists about sleep apnea risks with orthodontic procedures? Has that changed over the years? Did I permanently screw up my kid?

\section{Signed, \\ A Concerned Mother}

Of course, I wrote back to this mother explaining that OSAS is a neurogenic problem not an anatomic disease and there is no evidence, research or biologic rationale for such an association. Unfortunately, this type of irresponsible behavior by "dental professionals" is far too common. This situation reminds me of the 1980s when orthodontists "sold" orthodontic therapy as a treatment for temporomandibular joint dysfunction. It did not take long for some ambitious attorneys to make the connection that if changing the occlusion of the teeth "correctly" can treat TMD, then changing the occlusion of the teeth "incorrectly" can cause TMD. Now, thirty years and millions of dollars later, it is well documented that orthodontic alignment of teeth is neither the cause nor the treatment of TMD. This does not mean that dentists should not treat OSAS but rather that we stress to patients that OSAS is not caused by abnormal anatomy of the jaws and teeth. The cause is neurogenic. In the words of noted the philosopher, George Santayana* "Those who cannot remember the past are condemned to repeat it." So for those of you who intend to treat OSAS with dental appliances, "Caveate Emptor!".

It is critical therefore that guidelines of the American Academy of Sleep be followed that recommend "oral appliances should be fitted by qualified dental personnel who are trained and experienced in the overall care of oral health, the temporomandibular joint, dental occlusion and associated oral structures. Dental management of patients with OAs should be overseen by practitioners who have undertaken serious training in sleep medicine and/ or sleep related breathing disorders with focused emphasis on the proper protocol for diagnosis, treatment, and follow up" ${ }^{\prime 21}$.

A continuous positive airway pressure device applies continuous positive pressure to the upper airway through a mask that covers the nose

\footnotetext{
*George Santayana, The Life of Reason, Volume 1, 1905. US (Spanish-born) philosopher (1863-1952).
} 
and mouth. The level of pressure needed for each patient can be determined in a sleep laboratory. Because of the inconvenience of the device only forty-six percent of the patients had acceptable compliance with the device, defined as the use of the machine for more than four than hours per night for more than 70 percent of observed nights ${ }^{36}$. Despite poor compliance, patients treated with CPAP demonstrated improved neuropsychiatric function, reduced daytime sleepiness, and improved cardiac symptoms. In adults, CPAP is the initial treatment of choice, while in children the use of the device is limited to only those cases who did not respond well to adenotonsillectomy, or in whom this procedure is contraindicated $^{36}$. CPAP, however, is efficacious in children and remains a valid option.

Adenotonsillar hypertrophy is the major cause of obstructive sleep apnea in chidren ${ }^{33}$. Therefore, it is not surprising that adenotonsillectomy is the most frequently used treatment of sleep apnea in children ${ }^{15,17}$. In comparing children with sleep apnea before and after adenotonsillectomy, Stewart et al. ${ }^{39}$ found that after the procedure apnea-hypopnia index decreased significantly. Similarly, the quality of life scores, as seen in general health perception, behaVior and parental impact improved, and were much higher than in children with sleep apnea who did not have the surgery. Similarly, in a different study, children's behavior greatly improved in the areas of aggression, atypicality, depression, and hyperactivity after adenotonsillectomy ${ }^{25}$. The mean age of sleep apnea children in this study was 7 years.
Effectiveness of uvulopharyngopalatoplasty with genioglossal advancement was examined by Liu et al. ${ }^{23}$. Adult patients with pre-operative apnea/hypopnea index of greater than 40 were evaluated 3 months after the procedure. The overall success rate of $52 \%$ shows that this surgery is a viable option for patients who are unwilling to receive CPAP treatment. An alternative procedure in adults is uvulopalatal flap, which involves bilateral tonsillectomy, dissection and removal of submucosal adipose tissue of the soft palate, and repositioning of the flap ${ }^{24} .84 \%$ of patients with OSAS who underwent this surgery achieved good results, as defined by at least $50 \%$ reduction in RDI and improved oxygen saturation. 3-D CT scan of these patients also revealed a significant increase in retropalatal space.

Advancing the mandible forward is a useful procedure for patients with sleep apnea. Rachmiel et al. ${ }^{32}$ examined young patients (between 7 months and 12 years) who underwent bilateral mandibular distraction. CT scans revealed $72 \%$ increase in upper airway volume accompanied by improved apnea index, oxygen saturation, and elimination of OSAS symptoms. Conley et al. ${ }^{8}$ advocated a procedure involving transverse expansion of the maxilla and mandible using distraction osteogenesis, followed by mandibular and maxillary advancement to treat obstructive sleep apnea. As can be seen from this brief review, a wide spectrum of surgical options is available to sleep apnea patients. Soft tissue and skeletal surgery may be a viable alternative for patients who are unwilling to use CPAP Sleep testing showed that CPAP and surgery are equally 
effective in resolving obstructive sleep apnea ${ }^{7}$.

Mandibular advancing appliances vary greatly in design. Blanco et al. ${ }^{5}$ tested a removable soft elastic silicone positioner that created a $5-\mathrm{mm}$ vertical opening as well as mandibular advancement. After the 36-month therapy with the appliance patients with obstructive sleep apnea showed a mean reduction in $\mathrm{AHI}$ from 33 to 9.6; decreased Epworth Sleepiness Scale scores (used to measure daytime sleepiness); and decreased snoring. A Mandibular Advancement Splint was effective in reducing ESS scores and RDI scores in patients with sleeprelated breathing disorders ${ }^{20}$. Twentyone of the 26 subjects reported an $81 \%$ reduction in snoring and $86 \%$ of the subjects reported better quality of

\section{3 - SIDE EFFECTS}

The efficacy of using oral appliances in the management of OSAS has been documented in the literature. However, there are some inherent risks for undesirable effects for patients using dental devices every night to manage this chronic disease. These adverse effects fall into three categories.

- First, since teeth never become permanently attached to bone unless there is a bony ankylosis, any tooth born oral appliance can cause tooth movement. Ghazal et al. ${ }^{12}$, retrospectively examined the dental changes that occurred after patients had worn the Thornton Adjustable Positioner (TAP) for over two years (a device anchored on teeth in both arches and can therefore result in reciprocal forces leading to dental side sleep. Rose et al. ${ }^{35}$ tested efficacy of a two splint mandibular protruding appliance and also found positive results: the mean $\mathrm{AHI}$ was reduced from 19 to 3.3, and oxygen saturation showed improvement. $88 \%$ of the patients achieved the therapeuticallysignificant $\mathrm{AHI}$ of below 5. In a prospective, crossover study of 16 patients with sleep apnea ${ }^{22}$, Twin block and Herbst appliances were used in adults. Each subject used both appliances separated by a 2-week washout period. AHI, snoring, and oxygen saturation were measured. The results showed that there was no difference in performance of the two appliances. However, the Herbst appliance proved to be more popular with patients.

effects) ${ }^{12}$. Evaluating consecutively diagnosed and treated patients for OSAS, they compared the baseline findings with follow-up findings after more than 24 months of continuous appliance wear. The authors reported $24 / 47$ of the patients were wearing the splints regularly after an average of $33+/-9.1$ months. The primary dental changes noted included a significant reduction in overbite $(p=$ $0.006)$, a reduction in the frontal overbite of more than $1 \mathrm{~mm}$ in ten patients $(47.6 \%)$, and the overbite of one patient decreased by $4 \mathrm{~mm}$. The maxillary front teeth showed significant palatal tipping and those in the mandible significant labial tipping. However, the clinical significance of dento-alveolar changes can only be 


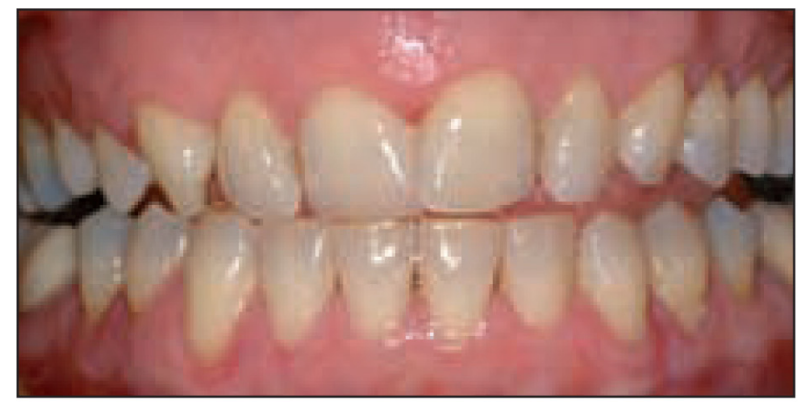

a

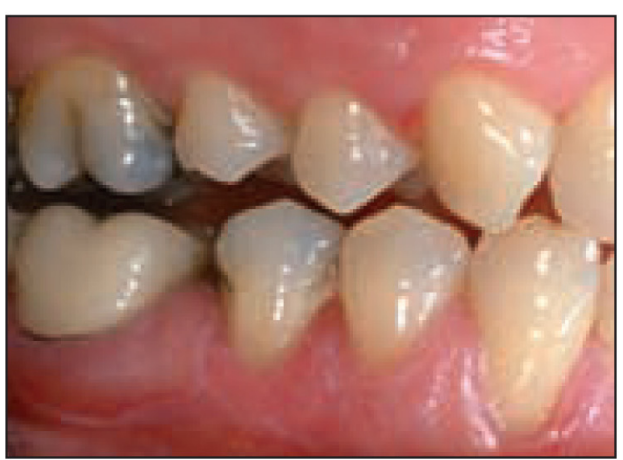

b

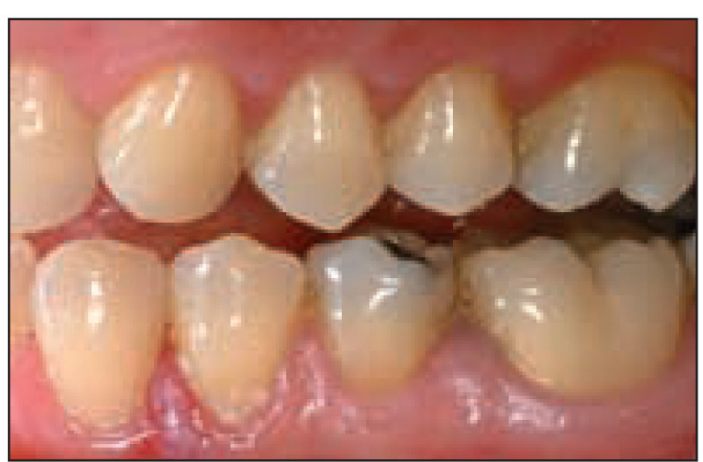

c

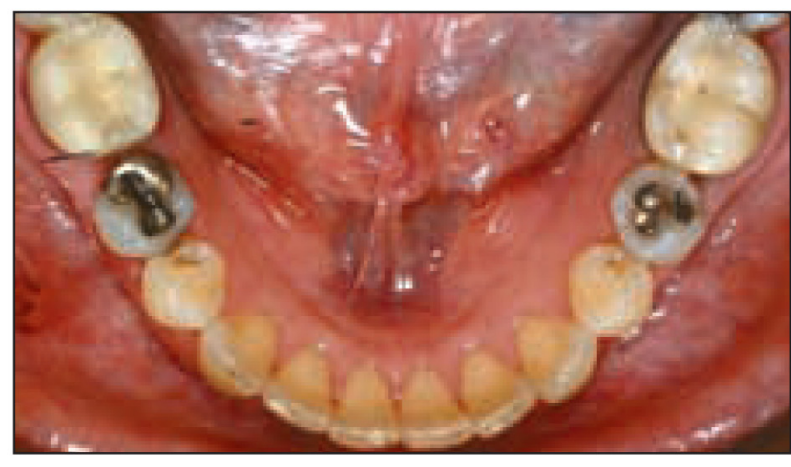

d

Figures 6 a to $d$

A patient treated by her general dentist used a dentally anchored device for 4 months that resulted in the occlusal insufficiency shown above. Orthodontic treatment was required to establish optimal occlusion. Please note open contacts mesial to the lowerfirst molars.

addressed by the dental specialists the patient. Figures 6 a to $d$ are of a patient treated with a dental device anchored to teeth in both arches. In a few months the dental changes created a masticatory deficiency that resulted in a resulted in a nervous dentist and concerned patient. The risk for tooth movement is directly related to two factors, the force placed on the teeth and the duration of the force. To minimize the risk of tooth movement we recommend the smallest amount of mandibular 


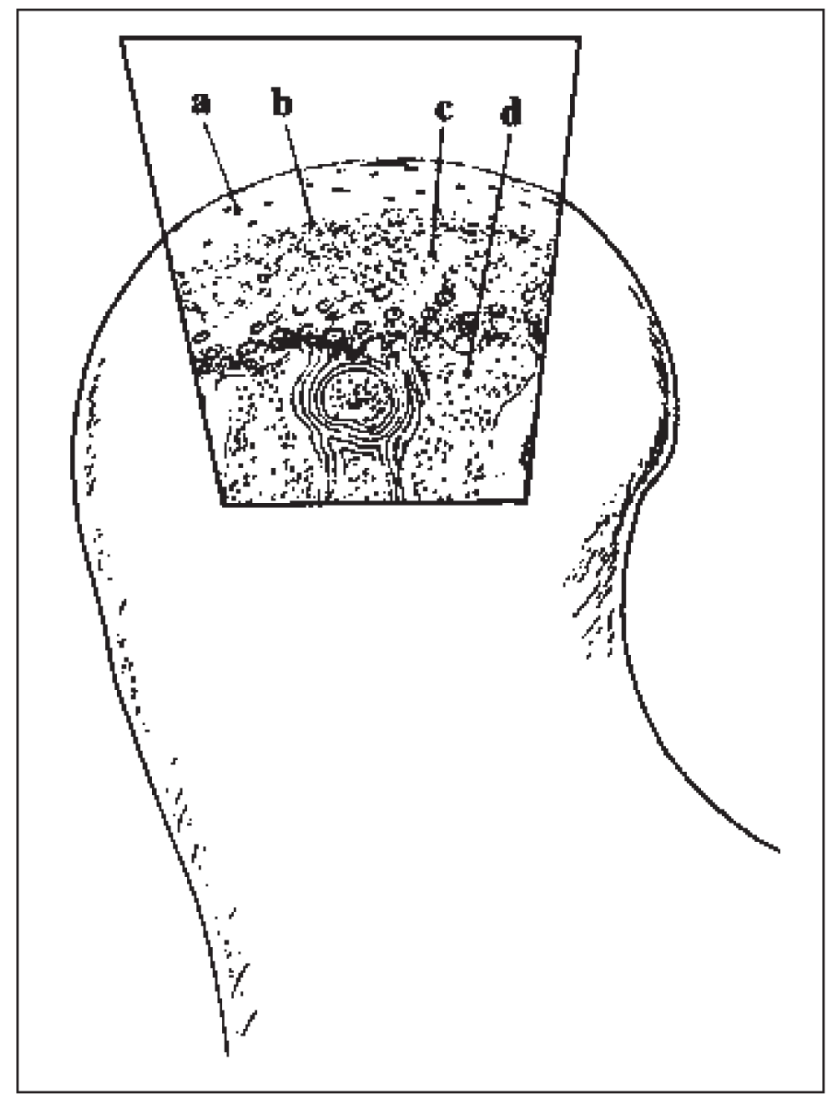

Figure 7

The different layers of the articular surface of the condyle:

a: vascularized connective tissue;

b: special layer of prechondroblast cells;

c: endochondral replacement by bone as the deeper layers advance;

$d$ : a trail of continually forming endochondral bone following the moving cartilage.

advancement possible. The limit the duration we recommend that the oral appliance be used in conjunction with the oral appliance used no longer than one week at a time. Following this protocol we have not observed tooth movement in our patients.

- The second undesired effect is on the tissues of the TMJ. Even an appliance worn for one night will result in an increase in synovial fluid within the joint. This increase in synovial fluid is significant for patients who have a very small difference in condylar position between centric relation and maximum intercuspation. For this patient group, the increase in synovial fluid results in a phenomenon whereby the patient cannot close the posterior teeth together following overnight use of the appliance. Patients report that this effect lasts from one to three hours. While this effect is transient, it is annoying to patients who are occlusally aware. 
- The third category of undesired effects is on the condyle. The primary role of the condyle is to maintain the articulation of the mandible with the neurocranium through the glenoid fossa. The condyle is also capable of adaptive and responsive growth and under normal circumstances can withstand the compressive forces of mandibular function. Thus the condyle (1) provides a pressureolerant articular contact, and (2) makes possible a multidimensional growth capacity in response to ever-changing, developmental conditions. A unique capsular layer of poorly vascularized connective tissue covers the articular surface of the condyle (fig. 7 a). This membrane is highly cellular early in development, but becomes densely fibrous with age and function. Just deep to it is a special layer of prechondroblast cells (b). This is the predominant site for cellular proliferation, and it is responsible for the tissue-feeding process providing an ongoing flow of new developing cartilage (layer c) for endochondral replacement by bone as the deeper layers advance. The proliferative process produces the "upward and backward" growth

\section{4 - CONCLUSION}

The awareness of obstructive sleep apnea syndrome has grown rapidly worldwide in the last decade. The etiology and sequela, symptoms and pathophysiology, and treatment modalities are all better understood. Among treatments commonly prescribed, oral appliances are rightfully gaining ground as an effective tool in the management of this serious disease. Our ability (as dentists and movement of the condyle. The condylar cartilage moves by prechondroblast cell divisions with an equal amount of cartilage removal at the cartilage-bone interface. The removal phase involves replacement with endochondral bone. A trail of continually forming endochondral bone thus follows the moving cartilage, as schematized by layer $d$. To monitor and minimize the deleterious side effects of appliances and monitor patient progress, the American Academy of Sleep Medicine recommends that patients treated with oral appliances be followed every 6 months for the first year, and at least annually thereafter $^{21}$. The purpose of follow up is to monitor patient adherence, evaluate device deterioration or maladjustment, evaluate the health of the oral structures and integrity of the occlusion, and assess the patient for signs and symptoms of worsening OSAS. The report recognizes that improper use of the device is a potential problem for patients using oral appliances and concludes that close communication between the patient, dental specialist and the referring physician is most conducive to good patient care ${ }^{21}$.

orthodontists) to contribute in the management of OSAS comes with a vital responsibility. Our responsibility starts with implementing educational modules aimed at creating dental practitioners who are not only qualified to insert and monitor oral appliances but who are competently trained in the areas of sleep related breathing disorders, the temporomandibular joints and dental occlusion. 
Additionally, it is important we evaluate the short and long term benefits/ limitations of oral devices. Issues to be considered will range from the costs of care to effectiveness of different appliances, and quality of life issues to life expectancy itself. Emerging technologies are likely to allow for superior and more efficient appliances and monitoring systems in the future. As the boundaries of dentistry and orthodontics are redefined and scope of our practices change, it is vital we take a close look at the areas we are growing into, sufficiently educate ourselves for the better of our patients and ultimately, our field of dentistry and specialty of orthodontics.

\section{REFERENCES}

1. Anonsen C. Laryngeal Obstruction and Obstructive Sleep Apnea Syndrome. Laryngoscope 1990;100:775-81.

2. Avior G, Fishman G, Leor A, Sivan Y, Kayshar N, Derowe A. The effect of tonsillectomy and adeoidectomy on inattention and impulsivity as measured by the test of variables of attention in children with obstructive sleep apnea syndrome. Otolaryngology - Head and Neck Surgery 2004;131(4):367-71.

3. Bacon WH, Turlot JC, Krieger J and Stierle JL. Cephalometric evaluation of pharyngeal obstructive factors in patients with sleep apneas syndrome. Angle Orthod 1990;60:115-21.

4. Beninati W, Harris CD, Herold DL, Shepard JW, Jr. The effect of snoring and obstructive sleep apnea on the sleep quality of bed partners. Mayo Clinic Proceedings. 1999;74(10):955-8.

5. Blanco J, Zamarron C, Abeleira MT, Lamela C, Suarez D. Prospective evaluation of an oral appliance in the treatment of obstructive sleep panea syndrome. Sleep Breath. 2005;9(1):20-5.

6. Carvalho LB, Prado LF, Silva L, deAlmeida MM, Lora MI, Prado GF. Cognitive dysfunction in children with sleep-disordered breathing. L Child Neurol 2005;20(5):400-4.

7. Colin W, Duval S. Surgical treatment of obstructive sleep apnea. AORN J 2005;82(3):372-8

8. Conley RS, Legan HL. Correction of severe obstructive sleep panea with bimaxillary transverse distraction osteogenesis and maxillomandibular advancement. Am J Orthod Dentofacial Orthop 2006;12(2):283-92.

9. DeBerry-Borowiecki B, et al. Cephalometric Analysis for Diagnosis and Treatment of Obstructive Sleep Apnea. Laryngoscope 1988;98:226-34.

10. El-Ad B, Lavie P. Effect of sleep apnea on cognition and mood. Int Rev Psychiatry 2005; 17(4): 277-82.

11. Ferguson AF, Fleetman JA. Consequences of sleep disordered breathing. Thorax 1995;50:998-1004.

12. Ghazal A, Jonas IE, Rose EC. Dental side effects of mandibular advancement appliances a 2-year follow-up. Orofac Orthop. 2008;69(6):437-47. Epub 2008 Nov 11.

13. Gonzal D, Pope DW. Snoring during early childhood and academic performance at ages thirteen to fourteen. Pediatrics 2001;107(6):1394-9.

14. Gozal D. Sleep-disordered breathing and school performance in children. Pediatrics 1998;108:616-20.

15. Guilleminault $\mathrm{C}$, Lee $\mathrm{JH}$, Chan A. Pediatric obstructive sleep apnea syndrome. Arch Pediatr Adolesc Med. 2005;159:775-85.

16. Hans MG, Nelson S, Luks VG, Lorkovich P, Baek SJ. Comparison of two dental devices for treatment of obstructive sleep apnea syndrome (OSAS). Am J Orthod 
Dentofacial Orthop. 1997;111(5):562-70.

17. Hoban TF. Obstructive sleep apnea in children. Curr Treat Options Neaurol. 2005;7(5):353-61.

18. Hu FB, Willett WC, Manson JE, et al. Snoring and risk of cardiovascular disease in women. Journal of the American College of Cardiology. 2000;35(2):308-13.

19. Ipsirogu OS, Fatemi A, Werner I, Tiefenthaler M, Urschitz MS, Schwartz B. Prevalence of sleep disorderes in school children between 11 and 15 years of age. Wien Klin Wochenschr 2001;113(7):235-44.

20. Johal A, Arya D, Winchester LJ, Venn PJ, Brooks H. The effect of a mandibular splint in subjects with sleep-related breathing disorders. Br Dent J 2005;199(9):591-6.

21. Kushida CA; Morgenthaler TI; Littner MR et al. Practice parameters for the treatment of snoring and obstructive sleep apnea with oral appliances: An Update for 2005. SLEEP 2006;29(2): 240-243.

22. Lawton HM, Battagel JM, Kotecha B. A comparison of the twin block and herbst mandibular advancement splints in the treatment of patients with obstructive sleep apnea: a prospective study. Eur J Orthod. 2005;27(1):82-90.

23. Liu SA, Li HY, Tsai WC, Chang KM. Associated factors to predict outcomes of uvulopharyngopalatoplasty plus genioglossal advancement for obstructive sleep apnea. Laryngoscope 2005;115(11):2046-50.

24. Li HY, Li KK, Chen NH, Wang CJ, Liao YF, Wang PC. Three-dimensional computed tomography and polysomnography findings after extended uvulopalatal flap surgery for obstructive sleep apnea. Am K Otolaryngol 2005;26(1):7-11.

25. Mitchell RB, Kelly J. Child behavior after adenotonsillectomy for obstructive sleep apnea syndrome. Laryngoscope 2005;115(11):2051-5.

26. Montgomery-Downs HE, Crabtree VM, Gozal D. Cognition, sleep and respiration in atrisk children treated for obstructive sleep apnea. Eur Respir J 2005;25(2):336-42.

27. Mulvaney SA, Goodwin JL, Morgan WJ, Rosen GR, Quan SF, Kaemingk KL. Behavior problems associated with sleep disordered breathing in school-aged children. J Pediatr Psychol 2005.

28. Nelson S, Kulnis R. Snoring and sleep disturbance among children from an orthodontic setting. Sleep and Breathing 2001;5(2):63-70.

29. Pae E-K. A Comparative Study of the Relationship between Airway Size, Tongue Activity, and Body Position. Master's Thesis, University of British Columbia. 1989.

30. Pracharktam N, Hans MG, Strohl KP, Redline S. Upright and Supine Cephalometric Evaluation of Obstructive Sleep Apnea Syndrome and Snoring Subjects. The Angle Orthod 1994;64(1):1-10.

31. Pracharktam N, Nelson S, Hans MG, Broadbent BH Jr, Redline S, Rosenberg K and Strohl KP. Cephalometric Assessment in Obstructive Sleep Apnea. Amer J Orthod Dentofacial Orthop 1996;109;4:410-419.

32. Rachimiel A, Aizenbud D, Pillar G, Srouji S, Peled M. Bilateral mandibular distraction for patients with compromised airway analysed by three-dimensional CT. Int J Oral Maxillofac Surg 2005;34(1):9-18.

33. Ray RM, Bower CM. Pediatric obstructive sleep apnea: the year in review. Curr Opin Otolorayngol Head Neck Surg. 2005;13(6):360-5.

34. Redline S, Tishler PV,Tosteson T, Williamson J, Kump K, Browner I, Ferret V and Krejci P. The Familial Aggregation of Obstructive Sleep Apnea. Am J Respir Crit Care Med 1995;151:682-7.

35. Rose CE, Germann M, Sorichter S, Jonas JE. Case control study in the treatment of obstructive sleep-disordered breathing with an intraoral protrusive appliance. J Orofac Orthop 2004;65(6):489-500.

36. Schechter MS. Technical report: diagnosis and management of childhood obstructive sleep apnea syndrome. Pediatrics 2002;109:1-17.

37. Shamsuzzaman AS, Gersh BJ, Somers VK. Obstructive sleep apnea: implications for cardiac and vascular disease. JAMA 2003;290(14):1906-14. 
38. Stauffer J, Clifford WZ, Zwillich W, et al. Pharyngeal Size and Resistance in Obstructive Sleep Apnea. Am Rev Respir Dis 1987;136:623-7.

39. Stewart MG, Glaze DG, Friedman EM, Smith EO, Bautista M. Quality of life and sleep study findings after adenotonsillectomy in children with obstructive sleep apnea. Arch Otolorayngol Head Neck Surg 2005; 131(4):308-14.

40. Stoohs R, Guilleminault C. Cardiovascular changes associated with obstructive sleep apnea syndrome. J. Appl Physiol 1992;72: 583-9.

41. Strelzow VV, Blanks RHI, Basie A and Strelzow AE. Cephalometric airway analysis in obstructive sleep apnea syndrome. Laryngoscope 1988;98:1149-58.

42. Strohl KP, Saunders NA, Fieldman NT, Hallett M. Obstructive Sleep Apnea in Familial Members. N Eng J Med 1978;229:969-73.

43. Strohl KP, Saunder NA, Sullivan CE. Sleep Apnea Syndromes. In: Saunders NA, Sullivan CE, eds Sleep and breathing. Vol 21. New York: Marcel, Dekker, Inc., 1984:365-401.

44. Strohl KP and Redline S. Recognition of obstructive sleep apnea. Am J Resp Crit Care Med 1996;154: 279-89.

45. Sullivan CE, Berthon-Jones $M$, Saunder NA. Pathophysiology of sleep apnea. In: Saunders NA, Sullivan CE, eds Sleep and breathing. Vol 21. New York: Marcel, Dekker, Inc., 1984:299-363.

46. Wilms D, et al. Anatomic Abnormalities in Obstructive Sleep Apnea. Ann Otol Rhinol Laryngol 1982;91:595-6.

47. Yaggi HK, Kernan WN, Lichtman JH, Brass LM, Mohsenin V. Obstructive sleep apnea as a risk factor for stroke and death. N Engl J. Med 2005;353(19):2034-41. 